\title{
Analysis of an Alanine/Arginine Mixture by Using TLC/FTIR Technique
}

\author{
Jun Liu, ${ }^{1,2}$ Feng-shan Zhou, ${ }^{3}$ Ran Guo, ${ }^{2}$ Ye Jiang, ${ }^{2,4}$ Xiaokun Fan, ${ }^{2,5}$ Anqi He, ${ }^{2}$ Yanjun Zhai, \\ Shifu Weng, ${ }^{2}$ Zhanlan Yang, ${ }^{2}$ Yizhuang $\mathrm{Xu}^{2}{ }^{2}$ Isao Noda, ${ }^{6}$ and Jinguang $\mathrm{Wu}^{2}$ \\ ${ }^{1}$ Department of Biochemistry, Wenshan University, Wenshan 663000, China \\ ${ }^{2}$ College of Chemistry and Molecular Engineering, Peking University, Beijing 100871, China \\ ${ }^{3}$ School of Materials Science and Technology, China University of Geosciences, Beijing 100083, China \\ ${ }^{4}$ College of Pharmacy, Liaoning University of Traditional Chinese Medicine, Shenyang 11660, China \\ ${ }^{5}$ College of Chemistry and Material Science, Hebei Normal University, Shijiazhuang 050024, China \\ ${ }^{6}$ Department of Materials Science and Engineering, University of Delaware, Newark, DE 19716, USA
}

Correspondence should be addressed to Fengshan Zhou; zhoufs@cugb.edu.cn and Yizhuang Xu; xyz@pku.edu.cn

Received 6 September 2013; Accepted 14 November 2013; Published 28 January 2014

Academic Editor: Edyta Proniewicz

Copyright (C) 2014 Jun Liu et al. This is an open access article distributed under the Creative Commons Attribution License, which permits unrestricted use, distribution, and reproduction in any medium, provided the original work is properly cited.

We applied TLC/FTIR coupled with mapping technique to analyze an alanine/arginine mixture. Narrow band TLC plates prepared by using $\mathrm{AgI}$ as a stationary phase were used to separate alanine and arginine. The distribution of alanine and arginine spots was manifested by a 3D chromatogram. Alanine and arginine can be successfully separated by the narrow band TLC plate. In addition, the FTIR spectra of the separated alanine and arginine spots on the narrow band TLC plate are roughly the same as the corresponding reference IR spectra.

\section{Introduction}

Thin-layer chromatography (TLC) is a widely used separation method and has been extensively applied in the analysis of various mixed samples $[1,2]$. In conventional TLC experiments, separated samples are identified by color, UV-Vis absorbance, or fluorescence signals. Thus, it becomes difficult for TLC in the analysis of samples that lack UVVis absorbance and fluorescence signals. Since visualization reagent may not be suitable for all substances with a broad range of molecular structures, the utility of visualization reagents for unknown TLC spot identification is somewhat limited. Moreover, UV-Vis and fluorescent signal provide rather limited information concerning molecular structure of separated spots. Thus, alternative spectroscopic method is needed to be applied after TLC separation. FTIR spectrum is a universal tool that can identify various organic substances without any requirement on UV-VIS absorbance or fluorescence signals. Additionally, FTIR spectrum provides a large amount of information relevant to functional groups of separated substances [3, 4]. Moreover, it might be possible to directly recognize unknown separated spots on TLC plate based on their FTIR spectra. In addition, the advancement in mapping/imaging techniques of FTIR microscope enables us to obtain FTIR spectra of every microregion on a TLC plate automatically. Thus, it has become possible to perform TLC/FTIR analysis in a quick and convenient manner.

Since the 1960s, some TLC/IR technique appeared in the literature $[5,6]$. In such an experiment, suitable solvents were used to transfer each separated analyte out of the TLC plate. IR spectrum of the obtained sample was recorded after removing the solvent by vaporization. The procedure is tedious and time-consuming, and the analysis is under a risk of sample loss and contamination. Later, Percival and Griffiths, Zuber et al., Lloyld et al., and White developed a more practical in situ TLC/IR technique [712]. This approach is quick, convenient, and free of the risk of sample loss since FTIR spectrum is directly obtained from the separated sample spot on a TLC plate. However, traditional TLC stationary phases, such as silica and alumina, exhibit a strong background absorption that can bring about significant interference to sample identification. Danielson et 
al. proposed to use zirconium oxide that has no absorbance in mid-IR region as a new stationary phase for TLC/FTIR analysis $[13,14]$. However, the problem of interference from stationary phase is not adequately addressed, since water adsorbed on zirconium oxide still brings about severe interference in the measurement of FTIR spectra. Consequently, there have been only few papers $[15,16]$ relevant to TLC/FTIR published around the world during the past decade.

We proposed to use IR-transparent, insoluble inorganic salt particles as novel stationary phases for TLC/FTIR analysis to solve the above problem. Since the stationary phase is transparent for infrared light, the interference caused by IR absorption from stationary phase is naturally removed. The inorganic salt should be insoluble so that the possibility of stationary phase being destroyed by mobile phase can be avoided. Controlling the size of the inorganic salt particles is helpful to improve the performance of TLC/FTIR analysis due to the following reasons. (1) Decreasing the size of stationary phase particles alleviates the effect of light scattering so that the quality of the corresponding FTIR spectra can be improved. (2) As the size of the particles of stationary phase decreases, the efficiency of TLC separation is enhanced. (3) Decreasing the size of the inorganic salt particle results in the increasing of the specific area of the stationary phase. Thus, more analytes are allowed to be adsorbed on the surface of the stationary phase. This advantage is helpful to alleviate the overloading problem and improve the sensitivity of detection of separated sample by using FTIR measurement.

Based on the above consideration, a systematic work on the TLC/FTIR analysis has been conducted in our laboratory in recent years [17-19]. Our experiments showed that barium fluoride and silver iodide particles may be used as new stationary phases for TLC/FTIR analysis. Experimental result demonstrated that $\mathrm{BaF}_{2}$ particles tend to retain significant amount of adsorbed water, while it is much easier to remove adsorbed water completely for silver iodide particles. Thus, we focus on the TLC/FTIR studies by using AgI particles as stationary phase.

In parallel, we have made considerable effort to develop new approach to prepare TLC plates. In the traditional way of the preparation of TLC plate, polymeric adhesive is utilized to bind stationary phase particles together. This is not suitable for TLC/FTIR analysis, since polymeric adhesive has strong absorbance in IR region and brings about significant interference in the FTIR detection. Recently, we propose a new technique to prepare narrow band TLC plates [20]. Experimental results demonstrate that the performance of TLC/FTIR is significantly improved by using the narrow band TLC plates.

To validate whether TLC/FTIR technique is applicable to real chemical system, we used narrow band TLC plate to separate alanine and arginine. Subsequently, FTIR microscope with mapping techniques was used to reveal the distribution of colorless alanine and arginine band on the narrow band TLC plate.

\section{Experimental}

All reagents are of AR grade. Alanine, arginine, n-butanol, formic acid, acetic acid, and silver nitrate were obtained from
Beijing Chemical Factory. Potassium iodide was a product of Sinopharm Chemical Reagent Co., Ltd.

Silver iodide was synthesized by a reaction between silver nitrate and potassium iodide. The experimental detail of the preparation of silver iodide particles can be found in our previous work [18]. The diameters of the silver iodide particles are around $100 \mathrm{~nm}$.

\section{Apparatus}

A Thermo-Fischer Nicolet iN10 MX FTIR spectrometer equipped with an IR microscope was used in the experiments.

\section{Procedures}

4.1. Preparation of Mixed Sample Solutions. $0.150 \mathrm{~g}$ alanine was dissolved in $10.0 \mathrm{~mL}$ formic acid to prepare a $1.5 \mathrm{wt} \%$ alanine solution. Similarly, $1.5 \mathrm{wt} \%$ arginine solution was prepared by dissolving $0.150 \mathrm{~g}$ arginine in $10.0 \mathrm{~mL}$ formic acid.

A mixed solution sample was prepared by mixing equal volumes of $1.5 \mathrm{wt} \%$ alanine solution and $1.5 \mathrm{wt} \%$ arginine solution together.

4.2. Narrow Band TLC Analysis. We prepared narrow band TLC plate to conduct the experiment. The preparation process of the narrow band TLC plates is described in detail in our previous work [20].

The sample solution was manually spotted at one end of narrow band TLC plate by using a glass capillary $(0.3 \mathrm{~mm}$ in diameter). After evaporation of solvent, the narrow band TLC plate was developed by using butanol/acetic acid/water mixture as a mobile phase (the volume ratio among butanol, acetic acid, and water is $8: 1: 1$ ). The typical development time was about 20-30 minutes.

4.3. In Situ TLC-FTIR Detection. Considering that the boiling temperature of butanol is quite high, the following pretreatment is performed: narrow band TLC plate after separation was first heated at $80^{\circ} \mathrm{C}$ for 4 hours. Then the temperature was elevated to $120^{\circ} \mathrm{C}$ and kept at that temperature for 4 hours. At last, the TLC plate was heated at $140^{\circ} \mathrm{C}$ for 4 hours. The above procedure is necessary to remove the residual mobile phase completely. Then TLC plates were examined directly by using the FTIR microscope. To reveal the distribution of alanine and arginine spots on the narrow band TLC plate, FTIR mapping technique under linear scan mode was utilized. That is to say, FTIR spectrum of every tiny region on TLC plate $(100 \mu \mathrm{m} \times 100 \mu \mathrm{m})$ is obtained sequentially along the direction of the diffusion of mobile phase. All the spectra were recorded under reflection mode at a resolution of $16 \mathrm{~cm}^{-1}$. To improve the signal-to-noise ratio, reflect spectrum from gold mirror was used as background spectrum.

$3 \mathrm{D}$ chromatogram that is utilized to reveal the distribution of alanine and arginine spots was generated via a program written in our lab by using the software of MATLAB. 


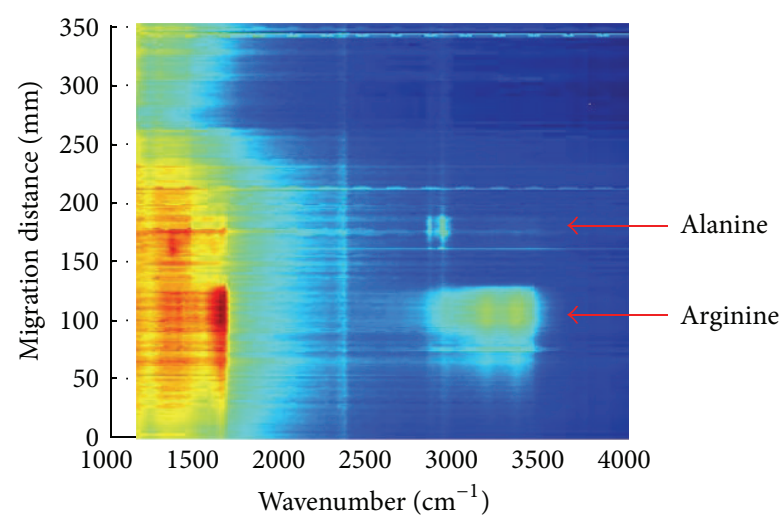

Figure 1: 3D chromatograph to characterize the distribution of arginine and alanine spots on a narrow band TLC plate.

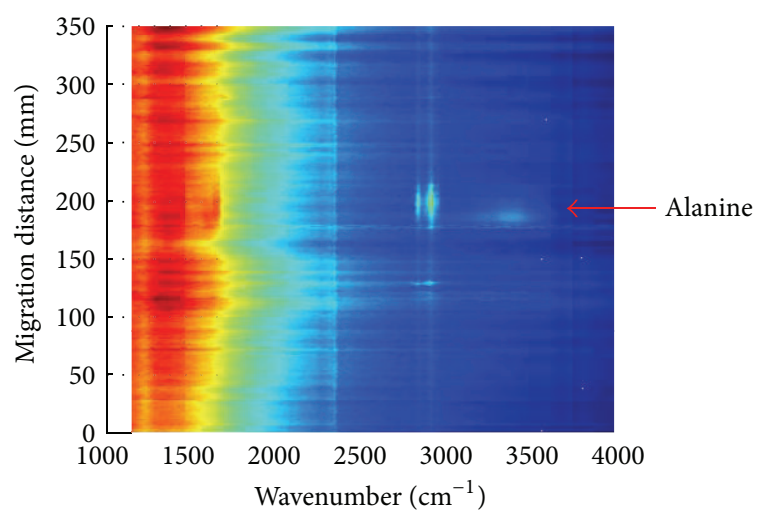

FIGURE 2: $3 \mathrm{D}$ chromatograph to locate the alanine spot on the narrow band TLC plate.

\section{Results and Discussion}

Since both alanine and arginine are colorless substances, no separated sample spot can be observed on the narrow band TLC plate. To reveal the distribution of alanine and arginine spots on the narrow band TLC plate, FTIR microscope with mapping technique is utilized. In the experiment, FTIR spectrum of each tiny region on the narrow band TLC plate is sequentially recorded. The obtained FT-IR spectra are organized along the direction of the diffusion of mobile phase so that a $3 \mathrm{D}$ chromatogram (the first dimension is wavenumber from FTIR spectrum, the second dimension is migration distance, and the third dimension is absorbance) is constructed. The $3 \mathrm{D}$ chromatogram is manifested via a contour map mode. As shown in Figure 1, two separated spots can be clearly visualized in the $3 \mathrm{D}$ chromatogram. To confirm that the above two spots are alanine and arginine, respectively, we used two solution samples that contain $1.5 \mathrm{wt} \%$ alanine only and $1.5 \mathrm{wt} \%$ arginine only for TLC/FTIR analysis. The experimental procedure is similar to those described previously. The resultant $3 \mathrm{D}$ chromatograms are shown in Figures 2 and 3 . Then we compare the FTIR spectra from the separated band shown in Figure 1 with the bands illustrated in Figures 2 and 3. Typical FTIR spectra of the two spots, which

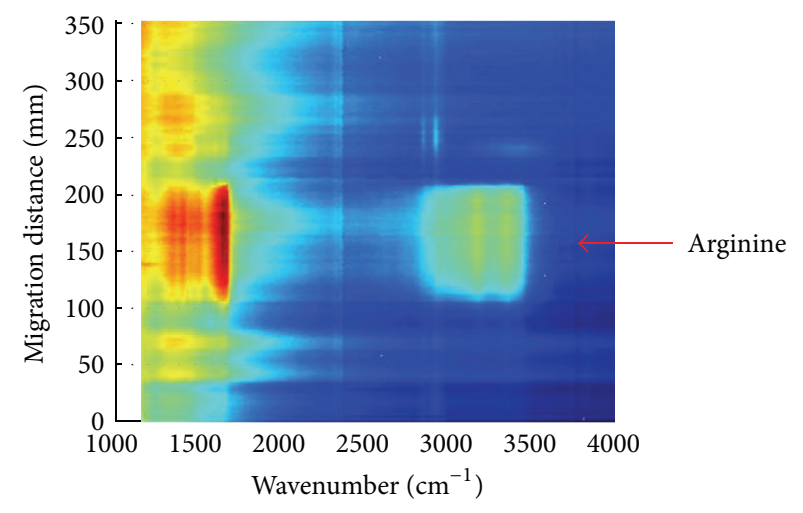

FIGURE 3: 3D chromatograph to locate the arginine spot on a narrow band TLC plate.

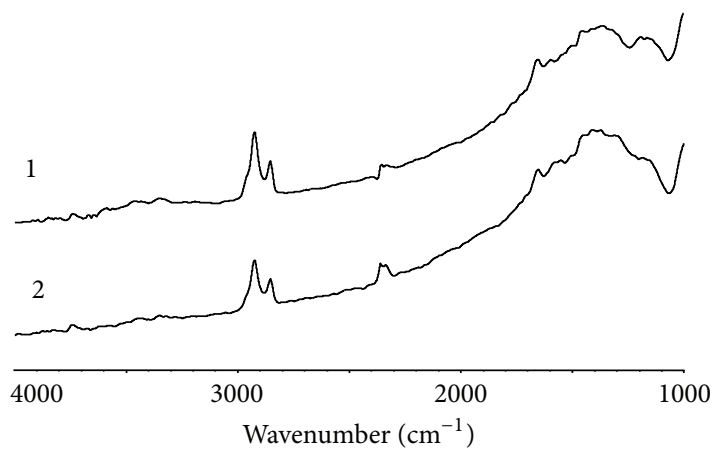

FIGURE 4: FTIR spectrum of alanine spot (trace 1) on a narrow band TLC plate. For comparison, a reference FTIR spectrum of pure alanine (trace 2) is also included.

are obtained by slicing the $3 \mathrm{D}$ chromatogram on the center of the sample spots, are shown as trace 1 in Figures 4 and 5 . FTIR spectra from the slices on the alanine and arginine spots shown in Figures 2 and 3 are used as reference spectra and displayed as trace 2 in Figures 4 and 5. FTIR spectra of upper and lower spot are almost the same as the reference spectra of alanine and arginine, respectively. These results confirm that the separated spots shown in Figure 1 are indeed alanine and arginine. It should be pointed out that alanine and arginine can accept proton or release proton as the $\mathrm{pH}$ changes. The variation of ionization states brings about drastic change in the corresponding FTIR spectra. On the other hand, alanine and arginine are often involved in complex hydrogen bond, which may bring about significant variation on the FTIR spectra. The above problems cause considerable difficulty on the spectral comparison between sample spectra and reference spectra. Thus, measuring the spectrum of separated spots and reference spectrum of corresponding standard material at the same experiment condition is very important to get reliable results.

\section{Conclusion}

In this work, we use narrow band TLC plates by using AgI as stationary phase to separate alanine and arginine. The 


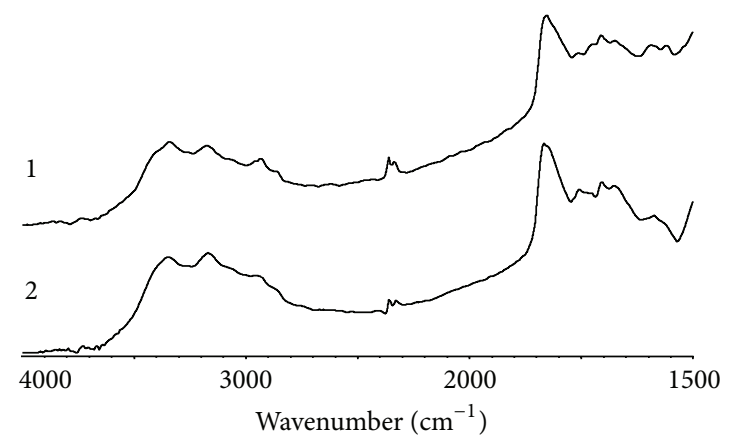

FIGURE 5: FTIR spectrum of arginine spot (trace 1) on a narrow band TLC plate. For comparison, a reference FTIR spectrum of pure arginine (trace 2) is also included.

distribution of alanine and arginine spots was probed by using FTIR microscope with spectral mapping technique and manifested by a 3D chromatogram. Experimental results demonstrate that alanine and arginine can be successfully separated by the narrow band TLC plate. Moreover, the FTIR spectra of the separated alanine and arginine spots on the narrow band TLC plate are roughly the same as the reference IR spectra of alanine and arginine. The present work demonstrates that TLC/FTIR technique is applicable in analysis complex mixtures.

\section{Conflict of Interests}

The authors declare that there is no conflict of interests regarding the publication of this paper.

\section{Acknowledgment}

This work is supported by the National Natural Science Foundation of China (51373003).

\section{References}

[1] M. Srivastava, Ed., High-Performance Thin-Layer Chromatography (HPTLC), Springer, Heidelberg, Germany, 2011.

[2] L. Y. He, Ed., Method and Application of Planar Chromatography, Chemical Industry Press, Beijing, China, 2005.

[3] J. G. Wu, Ed., Modern FTIR Spectroscopy Technology and Application, Science and Technology Press, Beijing, China, 1994.

[4] S. F. Weng, Ed., Fourier Transform Infrared Spectrometer, Chemical Industry Press, Beijing, China, 2nd edition, 2010.

[5] R. N. McCoy and E. C. Fiebig, "Technique for obtaining infrared spectra of microgram amounts of compounds separated by thin layer chromatography," Analytical Chemistry, vol. 37, no. 4, pp. 593-595, 1965.

[6] P. A. Sturm, R. M. Parkhurst, and W. A. Skinner, "Quantitative determination of individual tocopherols by thin layer chromatographic separation and spectrophotometry," Analytical Chemistry, vol. 38, no. 9, pp. 1244-1247, 1966.

[7] C. J. Percival and P. R. Griffiths, "Direct measurement of the infrared spectra of compounds separated by thin-layer chromatography," Analytical Chemistry, vol. 47, pp. 154-156, 1975.

[8] M. P. Fuller and P. R. Griffiths, "Diffuse reflectance measurements by infrared Fourier transform spectrometry," Analytical Chemistry, vol. 50, pp. 1906-1910, 1978.

[9] M. P. Fuller and P. R. Griffiths, "Infrared microsampling by diffuse reflectance Fourier transform spectrometry," Applied Spectroscopy, vol. 34, no. 5, pp. 533-539, 1980.

[10] G. E. Zuber, R. J. Warren, P. P. Begosh, and E. L. O’Donnell, "Direct analysis of thin-layer chromatography spots by diffuse reflectance Fourier transform infrared spectrometry," Analytical Chemistry, vol. 56, pp. 2935-2939, 1984.

[11] L. B. Lloyld, R. C. Yeates, and E. M. Eyring, "Fourier transform infrared photoacoustic spectroscopy in thin-layer chromatography," Analytical Chemistry, vol. 54, pp. 549-552, 1982.

[12] R. L. White, "Analysis of thin-layer chromatographic adsorbates by Fourier transform infrared photoacoustic spectroscopy," Analytical Chemistry, vol. 57, pp. 1819-1822, 1985.

[13] N. D. Danielson, J. E. Katon, S. P. Bouffard, and Z. Zhu, “Zirconium oxide stationary phase for thin-layer chromatography with diffuse reflectance fourier transform infrared detection," Analytical Chemistry, vol. 64, no. 18, pp. 2183-2186, 1992.

[14] S. P. Bouffard, J. E. Katon, A. J. Sommer, and N. D. Danlelson, "Development of microchannel thin-layer chromatography with infrared microspectroscopic detection," Analytical Chemistry, vol. 66, no. 13, pp. 1937-1940, 1994.

[15] W. He, R. Shanks, and G. Amarasinghe, "Analysis of additives in polymers by thin-layer chromatography coupled with Fourier transform-infrared microscopy," Vibrational Spectroscopy, vol. 30, no. 2, pp. 147-156, 2002.

[16] W. He, G. Cheng, F. Zao, Y. Lin, J. Huang, and R. Shanks, "Separation and identification of multicomponent mixture by thin-layer chromatography coupled with Fourier transforminfrared microscopy," Spectrochimica Acta A, vol. 61, no. 8, pp. 1965-1970, 2005.

[17] X. Liu, Q.-H. Pan, J. Ding et al., "Using barium fluoride fine particles as stationary phase for TLC/FTIR analysis," Spectroscopy and Spectral Analysis, vol. 31, no. 7, pp. 1767-1771, 2011.

[18] Q. Zhu, X. Su, H. J. Wu et al., "The analysis for silver iodide fine particles of TLC/FTIR matrix," Spectroscopy and Spectral Analysis, vol. 32, no. 7, pp. 1790-1794, 2012.

[19] W. Liu, H. J. Wu, X. P. Wang et al., "Preparation and application of barium fluoride particles as stationary phase for TLC-FTIR analysis," Chemical Journal of Chinese Universities, vol. 34, no. 6, pp. 1347-1352, 2013.

[20] F. Wang and H. J. Wu, "Development of narrow-band TLC plates for TLC/FTIR analysis," Analytical Methods, vol. 5, pp. 4138-4144, 2013. 

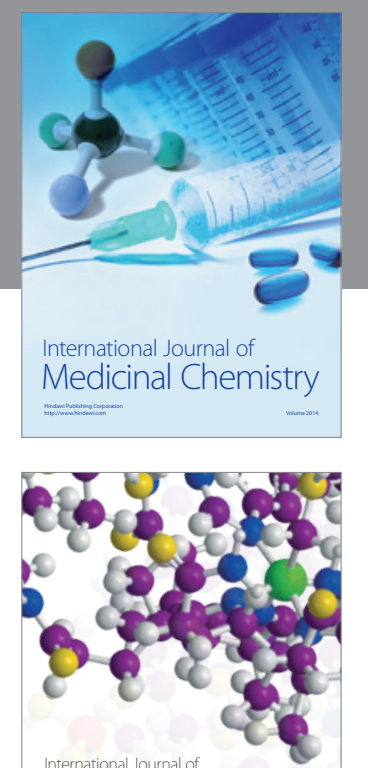

\section{Carbohydrate} Chemistry

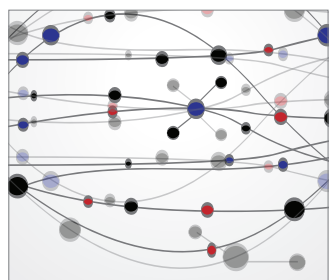

The Scientific World Journal
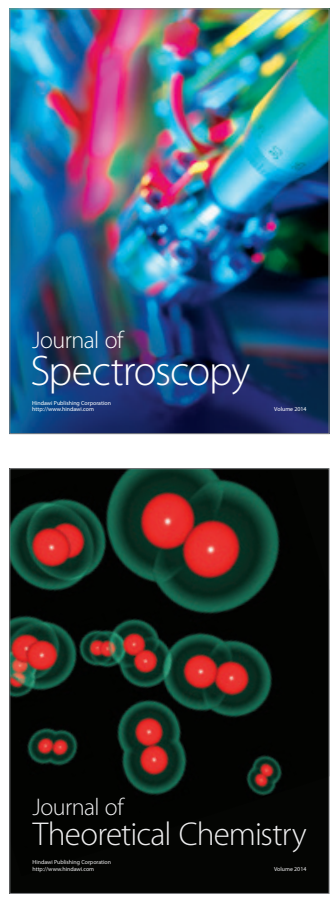
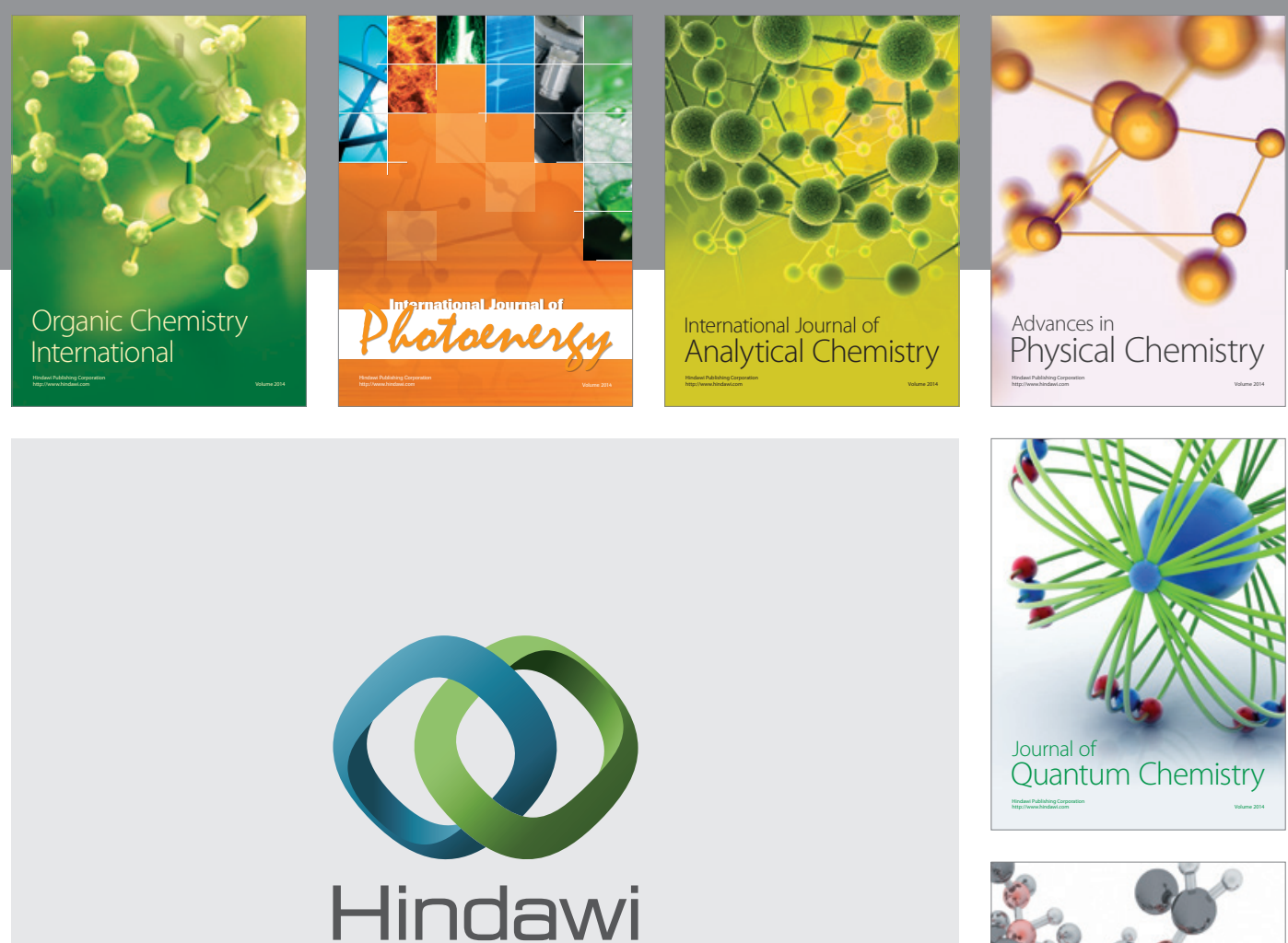

Submit your manuscripts at

http://www.hindawi.com

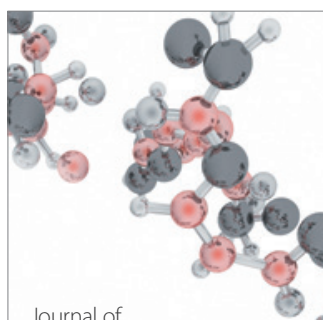

Analytical Methods

in Chemistry

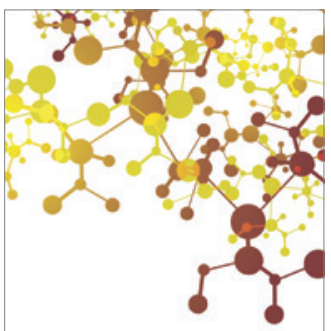

Journal of

Applied Chemistry

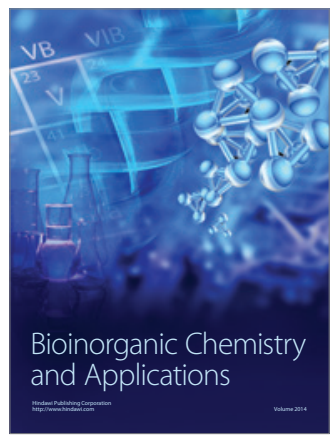

Inorganic Chemistry
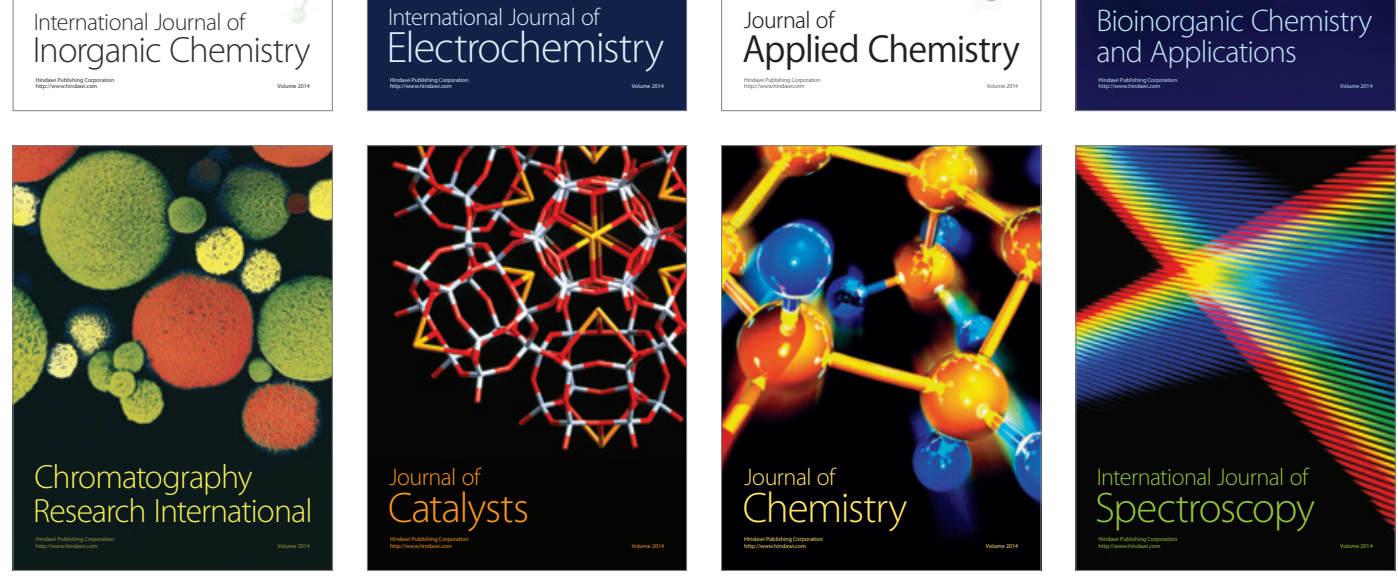